\title{
Planning for Global Precipitation Measurement
}

\author{
Gilbert Mark Flaming, \\ W. James Adams, Steven P. Neeck, and Eric A. Smith \\ National Aeronautics and Space Administration \\ Goddard Space Flight Center \\ Code 700.1 \\ Greenbelt, Maryland 20771 USA \\ Voice: (301) 286-7374 / Email: Gilbert.M.Flaming.1@gsfc.nasa.gov \\ Voice: (301) 286-2508 / Email: jim.adams@gsfc.nasa.gov \\ Voice: (301) 286-3017 / Email: Steven.P.Neeck.1@gsfc.nasa.gov \\ Voice: (301) 286-5770 / Email: Eric.Smith@pop900.gsfc.nasa.gov
}

\begin{abstract}
The Tropical Rainfall Measuring Mission (TRMM) has demonstrated the importance of rain measurements for both the science and the meteorological communities. The National Aeronautics and Space Administration (NASA) has recently established a Pre-Formulation Office to initiate the planning for Global Precipitation Measurement (GPM), a follow-on to TRMM. GPM is envisioned as a multi-satellite constellation using passive and active microwave sensors to take rainfall measurements every few hours on a global basis. This paper will discuss the programmatic status of GPM, and opportunities for international partnership arrangements. In addition, the top-level science requirements as currently identified, and concepts under consideration for obtaining the desired measurements, will be briefly discussed. Finally, ground data processing and distribution, and an overview of instrument concepts will be addressed.
\end{abstract}

\section{INTRODUCTION}

The November 1997 launch of the Tropical Rainfall Measuring Mission (TRMM) has demonstrated not only the feasibility of performing rainfall measurements from space, but has also demonstrated the value of those measurements for climate research and for weather monitoring and forecasting. TRMM was launched in November 1997 from Tanegashima Space Center, Japan, aboard an H-II rocket, and placed into a 350 kilometers, $35^{\circ}$ inclined orbit. The instruments of primary interest for measuring cloud dynamics and rainfall are the TRMM Microwave Imager (TMI) and the Precipitation Radar (PR). The TMI provides measurements of vertically integrated columns of liquid water. An active sensor, the PR, is able to penetrate and stratify cloud measurements into layers 250 meters thick by the use of range-gating and various radar signal processing techniques. These two instruments have provided valuable information regarding rainfall, cloud structure, and the distribution and motion of water within the cloud. Data assimilation experiments suggest that a new mission that extends measurement capabilities to a global basis, and performs the measurements at a high temporal rate, will have a number of benefits. The data from such a mission will help in developing a global understanding of the dynamics of clouds and precipitation, be useful for research on the global water cycle, and very importantly, improve the accuracy of weather forecasts, especially forecasts of severe weather such as hurricanes. It is the National Aeronautics and Space Administration's (NASA's) intent that the global measurement of precipitation becomes a long-term, systematic measurement.

\section{PLANNING FOR GLOBAL PRECIPITATION MEASUREMENT (GPM)}

As a result of the capabilities demonstrated by TRMM, the science community became a strong advocate for continued research into rainfall and related areas. Soon after its launch, scientists involved with rainfall, hydrology, the global water cycle, climatology, and other disciplines began discussing a rainfall measuring mission which would provide global measurements with revisit times as frequent as every three hours. In 1999, NASA's Goddard Space Flight Center (GSFC) established a Pre-Formulation Office for the purpose of providing a more careful examination of the requirements, structure, and programmatic support for such a mission. In April 2001, the President's budget included an allocation of funds for NASA to begin planning for global precipitation measurement. In support of the Administration's interest, NASA Headquarters has directed GSFC to conduct Advanced Studies of Global Precipitation Measurement (GPM). "These advanced studies are to be structured to facilitate an anticipated approval-to-proceed with formal formulation activities in the future". Concurrent with the PreFormulation activities, technology development efforts were also being undertaken. For example, NASA's Earth Science Technology Office (ESTO) provided funding to advance the development of several enabling technologies which may be incorporated into the Constellation satellites, including a Synthetic-Thinned Array Radiometer (STAR) [1]. The GPM Pre-Formulation Office is pursuing the philosophy that only mature, low-risk technology will be used on the mission; any technology developments will need to demonstrate this maturity prior to being incorporated into the mission. 
The initial planning for this new rainfall measurement mission is being directed to satisfy two top-level objectives: (1) develop a better understanding of the horizontal and vertical structure of clouds and rainfall, and (2) provide rainfall measurements on a frequent, global basis. NASA and its Japanese partner from TRMM, the National Aeronautics and Space Development Agency (NASDA), have put together a preliminary mission concept. In this concept, a Core satellite will be developed that will make the measurements necessary for scientists to advance their understanding of the dynamics of cloud structure and rainfall processes. This satellite will use the same type of instrumentation as used by TRMM, a precipitation radar and a passive microwave radiometer. The measurements from this satellite will be augmented with rainfall measurements made on a global basis by a constellation of up to eight additional satellites, thus providing a global revisit time of three hours, as requested by climate researchers and weather forecasters. To achieve this capability, GPM will coordinate the development and launch of several GPM-dedicated satellites, and will use data streams containing rainfall information provided by existing weather and environmental research satellites. A notional description of the GPM Core satellite and the GPM-developed Constellation satellites follow.

\section{GPM CORE SATELLITE}

The GPM Core satellite will be the keystone of the GPM Constellation. The satellite will be launched into a $65^{\circ}-70^{\circ}$ inclination, 400 kilometer orbit. It will be the primary source of scientific information regarding cloud structure and rainfall dynamics. The on-board instrumentation will consist of a Dual-frequency Precipitation Radar (DPR) and a passive, multi-channel microwave radiometer. The addition for GPM of a second operating frequency to the precipitation radar will allow a substantial increase, when compared to TRMM, in the understanding of the dynamics of rainfall within the structure of clouds. The calibration of the DPR will take place during over-flights of up to four, geographically diverse GPM ground calibration sites. Calibration of the DPR will also allow calibration of the passive microwave radiometer, and provide a means to transfer a calibration standard to rainfall measurements obtained from other satellites. While partnership discussions between NASA and NASDA are currently in progress, it is the expectation of the GPM PreFormulation Office that the DPR and the spacecraft launch will be provided by the Japanese, and the United States will provide the Core spacecraft bus and a multi-channel microwave radiometer. Although the specific characteristics of the microwave radiometer have not been defined at this time, the success of the TRMM mission and the TRMM Microwave Imager (TMI) provide an excellent baseline from which the planning will start.

\section{GPM CONSTELLATION SATELLITES}

In order to make rainfall measurements on a global basis every three hours, up to eight satellites will be required. Fortunately, rainfall information is contained in measurements that will be available from operational weather satellites such as DMSP and NPOESS, and environmental research satellites such as NASA's EOS Aqua and NASDA's ADEOS II and GCOM-B1. By using these satellites as a foundation for developing a constellation, it is possible for GPM to achieve a three-hour revisit time with the addition of three-to-five satellites developed under the auspices of GPM. These GPMdedicated Constellation satellites, as a baseline, will consist of a spacecraft bus and a multi-channel, passive microwave radiometer. The Constellation microwave radiometer may, or may not, be an instrument identical to the microwave radiometer flown on the Core. The Constellation satellites will be placed into Sun-synchronous, circular orbits at an altitude between 600 and 850 kilometers; nodal crossing times will be selected to provide the best augmentation possible to the coverage provided by the weather and research satellites. The GPM-developed Constellation satellites will be provided by NASA and its partners from Europe and elsewhere.

\section{ADDITIONAL INTERNATIONAL PARTICIPATION IN GPM}

NASA is encouraging foreign participation in GPM. In order to provide a forum to discuss the opportunities for foreign participation, a GPM Planning Workshop was held May 2001. Over four hundred invitations were sent to national space agencies and scientists throughout the world. Partnering with foreign countries is important to NASA, not only for distributing the cost of the program, but to also engage foreign scientific expertise and to gain access to unique foreign facilities and resources. For example, the scientists involved with the planning for GPM have expressed a desire to have several, heavily instrumented, ground calibration sites for the Core spacecraft. These sites need to be located in a variety of environments, such as tropical locations with heavy rainfalls, coastal temperate regions, and semi-arid regions. A foreign contribution of ground calibration sites may provide access to physical environments that are not available in the United States, or otherwise available to the program. Foreign partners may also participate in other aspects of the program, perhaps by providing passive microwave sensors, assisting with the development of science algorithms, or providing relevant data streams from instruments used for other programs. It should be noted that NASA considers participation in the development and implementation of the measurement program to be a key requisite for inclusion in the science planning and analysis activities. Fig. 1 illustrates a notional development of the GPM Constellation over time. The top axis shows the reduction in Global Revisit Time as the number of constellation satellites increases. As may be seen, achieving short Global Revisit Time requires the support of our International partners. 


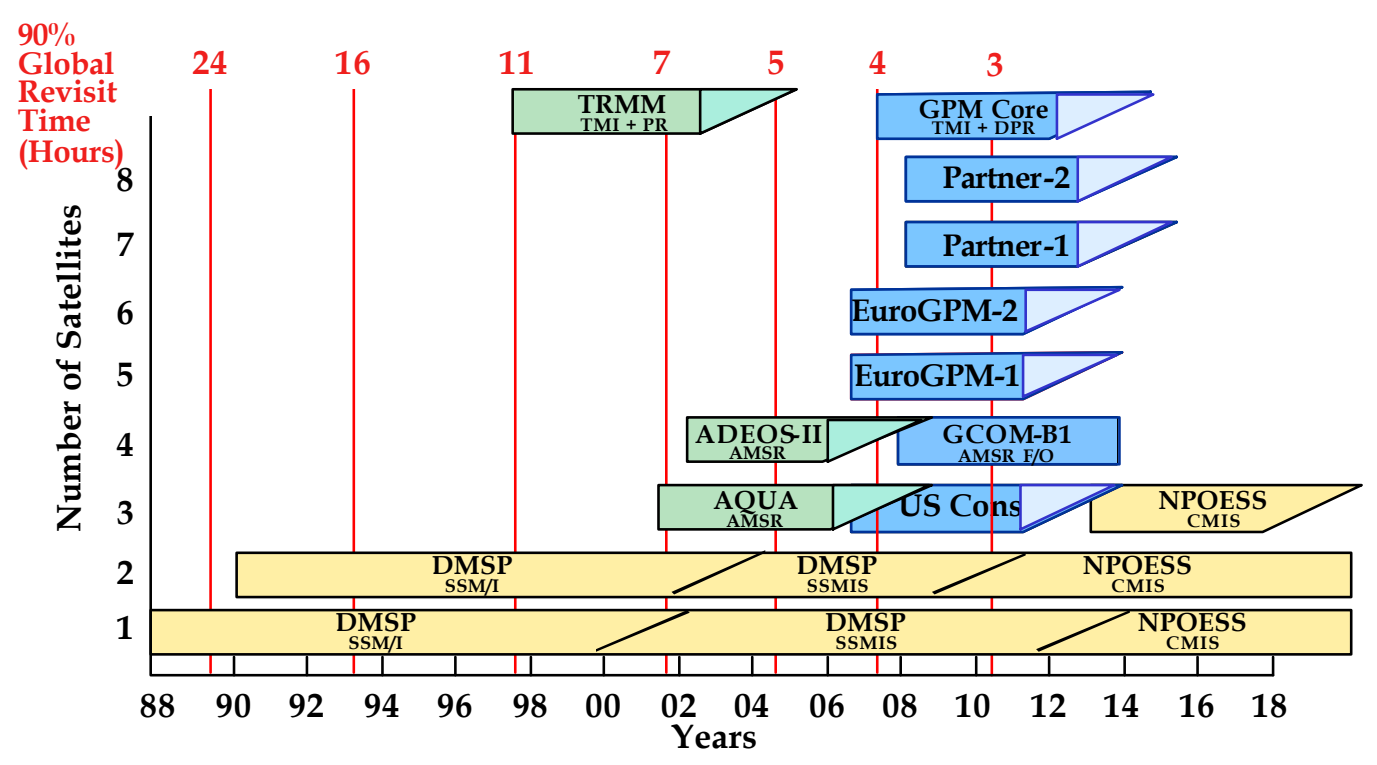

Fig. 1. Evolution of the GPM Satellite Constellation

\section{GROUND DATA PROCESSING AND DISTRIBUTION}

NASA has substantial experience in the development and operation of science data processing and distribution systems. For example, the TRMM Science Data and Information System (TSDIS) is a system developed and managed for the TRMM project by GSFC's Global Change Data Center Division. TSDIS was ready to process on the first day data was available, and has been operating successfully since then. Working with the TRMM Science Team, TSDIS is currently implementing algorithms to merge near-real time TRMM and SSMI data, a process that will also serve as a precursor for the merger of data from the multiple satellites and instruments of the GPM Core and Constellation. The GPM Project will implement a dedicated system for Global Precipitation Measurement. This system will capitalize upon the Agency's experience with the development of data system architectures, and will incorporate the emerging New Data and Information Systems and Services (NewDISS) NASA Standards. These activities will be managed as part of the GPM Project at NASA's Goddard Space Flight Center.

\section{PROGRAM PlAN AND SCHEDULE}

GPM has been in Pre-Formulation for the past two years, and will move into Advanced Studies this year. The Formulation Phase will extend from calendar year 2002 until early 2003 when the Preliminary Design Review (PDR) is planned. The Development Phase of the Program will start following PDR, and will continue until 2007, when both the Core and the first GPM-developed Constellation satellites will be launched. Following launch, a three-to-five year measurement campaign will take place. Initial discussions with NASDA, and with potential international partners, indicate that this schedule is compatible with their planning.

\section{CONCLUSION}

In conclusion, TRMM has demonstrated that a space-borne platform can be used for obtaining information regarding cloud dynamics and accurate rainfall measurements. The successes of TRMM have led to the establishment of an office at the Goddard Space Flight Center responsible for overseeing the development of a program to make long-term, systematic measurement of precipitation on a global basis. The planning for this activity is currently in progress.

\section{REFERENCES}

[1] Ruf, C.S., C.M. Principe, and S.P. Neeck, "Enabling Technologies to Map Precipitation with Near-Global Coverage and Hour-Scale Revisit Times," Proc. of the 2000 International Geoscience and Remote Sensing Symposium, Honolulu, HI, IEEE Cat. \#99CH37120, Vol. VII, 2988-2990, July 2000. 